\title{
Protéines du lait et nutrition des différentes catégories de population
}

\author{
E Grasset
}

\author{
Roussel Uclaf nutrition, Tour Roussel-Hoechst, 1, Terrasse Bellini, 92800 Puteaux, France
}

\begin{abstract}
Résumé - Une population comprend des individus très divers : des enfants et des personnes âgées, des sujets bien portants qui veulent, autant que possible, le rester et des malades qu'il ne faut pas aggraver mais, si possible améliorer. Comment évaluer l'adaptation des protéines laitières, particulièrement en nutrition clinique et dans des populations aussi diverses ? Sur un plan réglementaire, il n'y a pas d'accord général sur la méthode d'évaluation globale de la qualité d'une protéine. En France, cette qualité est évaluée à partir de l'aminogramme comparé à une référence, l'acide aminé limitant permet de calculer l'indice chimique. Aux USA, la FDA s'appuie sur une analyse in vivo (mesure du Protein Efficiency Ratio (PER), gain de poids du rat en croissance). Les 2 méthodes sont parfois loin d'être superposables, surtout pour l'homme malade. II existe de nouvelles méthodes d'étude du métabolisme protéique aussi bien in vitro que in vivo. L'utilisation des isotopes stables non radioactifs représente un apport très important. Le métabolisme protéique peut être évalué de manière globale (leucine) mais certains éléments méritent d'être étudiés séparément (glutamine). À ce sujet, nous avons besoin de données analytiques. L'étude de l'effet d'un nutriment protéique sur un paramètre fonctionnel ne peut pas faire abstraction des autres composants de la ration alimentaire. Comme pour le pétrole, l'industrie agro-alimentaire offre maintenant des possibilités de "cracking" des composants et permet des réponses nouvelles aux besoins de populations normales ou malades en modifiant les formes moléculaires, les compositions, et les apports en minéraux ou autres nutriments associés.
\end{abstract}

\section{diététique / glutamine / nutrition / peptide / protéine laitière}

Summary - Milk proteins and nutrition of different population subgroups. A population is made up of a wide variety of individuals: children and the elderly, the healthy and others who are ill and whose condition should not be aggravated, but if possible improved. The question is raised of how the adequacy of dairy proteins can be evaluated for such a diverse population with a particular emphasis on clinical nutrition, and as regards regulations, there is no general agreement on a single method for evaluating protein quality. In France, this quality is quantified by chemical index calculation. Amino acid content is compared to that of a reference protein. In the USA, the FDA relies on an in vivo evaluation of protein efficiency ratio, PER (weight gain of growing rats). Both methods are far from being similar, especially as regards their application to human diseases. New methods are now available for investigation of protein metabolism, both in vitro and in vivo. Use of stable non radioactive isotopes constitutes an important step. Protein metabolism can be evaluated by an index such as leucine turnover. The value of special components warrants separate evaluation. Glutamine is a good example, being a fuel which supplies both nitrogen and energy. Its importance in gut metabolism is now recognized. Due to analytical difficulties, glutamine content in protein and peptides has not been well determined. Analytical data are urgently required. The effect of quantity and nature of protein intake is actively studied in pathologic conditions. Severely ill patients may benefit from an adapted protein source with special amino acid contents or predigested peptides. Study of the effect of a single nutrient on functional parameters cannot usually be fully evaluated without considering the whole diet. The agro-food industry, like the petrol industry, can now isolate new components and 
provide new answers to nutritional requirements in both normal and pathological conditions. Molecular size, composition and associated nutrients and minerals can now be modified.

diet therapy / glutamine / nutrition / peptide / dairy protein

\section{INTRODUCTION}

Le lait constitue le premier aliment du nourrisson. Avec ses dérivés, il reste ensuite largement consommé. Les protéines laitières étant abondantes et de bonne valeur biologique, elles ont servi de matière première pour les régimes alimentaires des enfants ou des adultes malades ou devant suivre un régime particulier. La population est variée, les possibilités techniques sont également de plus en plus larges. L'ensemble devrait permettre de répondre aux besoins de la population. Encore faut-il disposer de critères d'appréciation.

\section{DÉFINITION DES CATÉGORIES DE POPULATION}

La France compte environ 55,6 millions d'habitants. 770000 nouveau-nés par an ont une espérance de vie de 80,3 ans pour les filles et de 72 ans pour les garçons. En Europe, en l'an 2000, 19,8\% de la population devrait avoir 60 ans ou plus (soit 99,8 millions de personnes). Certaines affections chroniques sont maintenant prises en charges : par exemple, en France pendant l'année 1986, 16 00017000 patients étaient traités pour une insuffisance rénale terminale, 1700 personnes vivaient grâce à un rein greffé fonctionnel et 1300 patients ont reçu un nouveau greffon (OCDE, 1990).

Ces populations diverses peuvent être définies ainsi.

\section{Populations normales}

Des conditions physiologiques variables définissent les individus d'une population normale. Des recommandations nutritionnelles spécifiques ont été formulées tenant compte de ces conditions (Dupin, 1981; National Research Council, 1989) et sont en partie résumées dans le tableau I.

\section{Age}

La plupart des normes distinguent des tranches d'âge de 3 mois en 3 mois pendant les premiers 6 mois de la vie, une tranche de 7 à 12 mois, puis des tranches de 2 à 3 ans jusqu'à la fin de l'adolescence, les besoins exprimés en $\mathrm{g} / \mathrm{kg}$ diminuant avec l'âge. Les aliments diététiques infantiles bénéficient d'une législation spécifique. II s'agit d'un marché assez important dépassant un milliard de francs par an.

Les personnes âgées posent un problème plus particulier. Alors que pour le développement de médicaments, des études ou des analyses particulières à cette population sont recommandées, pour le moment, il ne semble pas y avoir d'accord sur la définition d'une tranche d'âge ayant des besoins nutritionnels très différents de ceux des sujets plus jeunes.

\section{Grossesse, lactation}

De même que, lors de la croissance chez l'enfant, il existe des besoins particuliers. 
Tableau I. Apports quotidiens recommandés 1. Recommended dietary allowances ${ }^{1}$.

\begin{tabular}{llll}
\hline Age & Sexe & $\begin{array}{c}\text { Apport recommandé } \\
(g / \mathrm{kg})\end{array}$ & $(\mathrm{g} / \mathrm{j})$ \\
& & & \\
\hline $0-0,5$ & $\mathrm{M} / \mathrm{F}$ & 2,2 & 13 \\
$0,5-1$ & $\mathrm{M} / \mathrm{F}$ & 1,6 & 14 \\
$1-3$ & $\mathrm{M} / \mathrm{F}$ & 1,2 & 16 \\
$4-6$ & $\mathrm{M} / \mathrm{F}$ & 1,1 & 24 \\
$7-10$ & $\mathrm{M} / \mathrm{F}$ & 1,0 & 28 \\
$11-14$ & $\mathrm{M}$ & 1,0 & 45 \\
$15-18$ & $\mathrm{M}$ & 0,9 & 59 \\
$19-24$ & $\mathrm{M}$ & 0,8 & 58 \\
$24-50$ & $\mathrm{M}$ & 0,8 & 63 \\
$51+$ & $\mathrm{M}$ & 0,8 & 63 \\
$11-14$ & $\mathrm{~F}$ & 1,0 & 46 \\
$15-18$ & $\mathrm{~F}$ & 0,8 & 44 \\
$19-24$ & $\mathrm{~F}$ & 0,8 & 46 \\
$24-50$ & $\mathrm{~F}$ & 0,8 & 50 \\
$51+$ & $\mathrm{F}$ & 0,8 & 50 \\
Grossesse & & & \\
Allaitement & & \multicolumn{2}{c}{+10} \\
(premiers 6 mois) & & \\
\hline
\end{tabular}

${ }^{1}$ National Research Council, 1989.

L'augmentation des apports conseillés est maximale au cours du dernier trimestre de la grossesse et pendant l'allaitement (Dupin, 1981; National Research Council, 1989).

\section{Populations présentant des risques particuliers}

La plupart des habitants des pays développés consomment plus de protéines que l'apport minimal recommandé. Cet apport dépassant les besoins est excrété sous forme d'urée, synthétisée par le foie est excrétée par le rein. II existe donc des populations chez qui ceci peut être dangereux : les patients avec insuffisance hépatique (accumulation d'ammoniac en cas de charge protéique massive et d'atteinte hépatique très grave) et surtout insuffisance rénale. Dans ce dernier cas outre l'accumulation d'urée, les protéines alimentaires pourraient aggraver l'atteinte de la fonction rénale (cf ci-dessous).

Enfin, il existe un groupe de malades rares, mais qui a conduit à une meilleure connaissance des besoins en acides aminés et du métabolisme protéique : les personnes souffrant d'anomalies métaboliques héréditaires. Les symptômes de 2 de ces maladies moins rares que les autres, sont rappelés dans le tableau II. Du fait d'une anomalie enzymatique, il faut donner une alimentation artificielle comportant juste assez de l'acide aminé incriminé (sinon la croissance s'arrête), mais pas trop (sinon il s'accumule puisqu'il ne peut pas être métabolisé).

\section{COMMENT ÉVALUER L'ADÉQUATION AUX BESOINS}

Les apports actuellement recommandés ont été calculés, soit par des méthodes factorielles (pertes obligatoires et besoins

Tableau II. Anomalies héréditaires du métabolisme des acides aminés (exemples).

Hereditary anomalies of amino acid metabolism (examples).

\begin{tabular}{lll}
\multicolumn{1}{c}{ Maladie } & \multicolumn{1}{c}{$\begin{array}{c}\text { Acide aminé } \\
\text { accumulé }\end{array}$} & \multicolumn{1}{c}{$\begin{array}{c}\text { Enzyme } \\
\text { manquante }\end{array}$} \\
\hline $\begin{array}{l}\text { Phénylcétonurie Phénylalanine Phénylalanine } \\
\text { - hydroxylase }\end{array}$ \\
$\begin{array}{l}\text { Leucinse } \\
\text { «maladie du } \\
\text { sirop d'érable» }\end{array}$ & $\begin{array}{l}\text { AA branchés : Cétoacides- } \\
\text { Valine } \\
\text { Isoleucine }\end{array}$ \\
&
\end{tabular}


de renouvellement + marge de sécurité + besoins de croissance selon l'âge) soit par des études de bilan en conditions réelles, pas toujours disponibles et parfois contradictoires (Munro et Crim, 1988). La nutrition clinique considère également des patients malades avec des besoins augmentés ou chez qui un déficit nutritionnel antérieur doit être corrigé. L'évaluation clinique des produits de nutrition a ses spécificités propres (Desjeux et al, 1990).

\section{Réglementation actuelle}

La définition d'un apport protéique conduit à envisager 2 paramètres : la quantité apportée, la qualité. Pour définir la qualité, il existe 2 types d'approches établies, soit d'après les données analytiques : «indice chimique", soit par mesure comparative de la croissance in vivo : coefficient d'épargne protéique, CEP ou «PER» (Protein Efficiency Ratio) des anglo-saxons.

\section{Indice chimique}

C'est le critère règlementaire le plus important en Europe. L'aminogramme $(\mathrm{mg} / \mathrm{g}$ de protéine) de la protéine étudiée est comparé à celui d'une protéine de référence. Le rapport de la concentration de l'acide aminé limitant par rapport à celle contenue dans la protéine de référence est retenu pour calculer l'indice chimique exprimé en pourcentage. La protéine de référence dépend de la population étudiée et en particulier de l'âge. Tous les acides aminés n'interviennent pas. Le tableau III reproduit les références retenues pour :

- les aliments diététiques infantiles;

- les aliments destinés à une alimentation particulière (typiquement les mélanges nutritifs donnés par sonde ou par voie orale chez les adultes malades sous contrôle d'un médecin ou d'une diététicienne).
Tableau III. Indice chimique : références ( $g / 100$ g protéine)

Chemical index : references ( $g / 100 \mathrm{~g}$ protein)

AA Aliments diététiques
et de régime
de l'enfance 1 $\begin{gathered}\text { à une alimentation destinés } \\ \text { particulière }\end{gathered}$

$\begin{array}{lll}\text { lle } & 4 & 4 \\ \text { Leu } & 8,6 & 7 \\ \text { Lys } & 6,7 & 5,5 \\ \text { Met + Cys } & 2,9 & 3,5 \\ \text { Phe + Tyr } & 6,6 & 6 \\ \text { Thr } & 4,4 & 4 \\ \text { Trp } & 1,7 & 1 \\ \text { Val } & 4,5 & 5\end{array}$

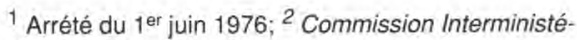
rielle, 1990.

${ }^{1}$ Decree of June 1st, 1976; ${ }^{2}$ Interministerial Commission, 1990.

\section{Coefficient d'épargne protéique (CEP ou PER)}

C'est la méthode retenue par la Food and Drug Administration américaine pour l'étiquetage nutritionnel des aliments (Food and Drug Administration, 1988). Si le PER est supérieur ou égal à celui de la caséine, la référence journalière recommandée est de $45 \mathrm{~g}$. Ainsi une dose d'un produit contenant $11,25 \mathrm{~g}$ de protéine apportera officiellement $25 \%$ des apports quotidiens recommandés (RDA). Si le PER est inférieur à celui de la caséine, elle devient de $65 \mathrm{~g}$. La même unité de l'aliment n'apporterait plus que $17 \%$ de l'apport recommandé. Si le PER est inférieur à $20 \%$ de celui de la caséine, le nutriment ne contribue pas à l'apport protéique. Le CEP est égal au rapport du gain de poids obtenu en 4 semaines sur la quantité de protéines ingérées chez des rats en croissance, constituant des groupes homogènes de poids initiaux identiques. Pour que la quali- 
té de la protéine soit limitante, l'apport doit être faible et ne représenter que 8-10\% de l'apport énergétique.

\section{Avantages et limites des deux méthodes}

L'indice chimique a le mérite de la simplicité. II est contrôlable par l'analyse. II ne tient pas compte de la digestibilité ni de l'absorption digestive.

Le CEP mesure un résultat global final in vivo. II est plus proche de l'effet physiologique. II est complexe à mesurer. II est réalisé avec un régime plus limité en protéines que l'alimentation occidentale courante. II ne mesure que le gain de poids et pas la rétention protéique réelle. II ne tient pas compte du remplacement des tissus. Le coefficient d'utilisation protéique net (NPU), avec groupe protéiprive et mesure de la rétention azotée, tient compte de ces 2 facteurs. Les protéines laitières sont plutôt avantagées par la méthode américaine puisque le CEP des protéines du lait est a priori supérieur ou égal à celui de la caséine (le CEP des protéines du lactosérum est supérieur à celui de la caséine). Par contre, l'indice chimique peut être amélioré de manière simple par une supplémentation en acides aminés libres (pour le tryptophane, cette possibilité vient d'être réglementairement limitée à certaines catégories de produits de nutrition).

Les 2 approches peuvent donner des résultats différents : comparant, chez le rat, l'administration de mélanges à base de petits peptides à des mélanges à base d'acides aminés libres de même anninogramme, donc d'indice chimique identique, Monchi et al (1991) ont trouvé une différence importante quant au NPU. Cette approche comparative reste malheureusement assez rare. Les nutritionnistes ont besoin de plus de données de ce type.

\section{Nouvelles méthodes d'investigation du métabolisme protéique}

Les mesures d'absorption se sont sophistiquées.

Les méthodes de mesure in vitro permettent d'étudier des vésicules de bordures en brosse isolées, des fragments de muqueuses isolées placées à plat dans une chambre de Ussing. Elles ont pu, par exemple, être appliquées à l'étude des anomalies congénitales de l'absorption des acides aminés.

Les mesures d'absorption in vivo sont réalisées à l'aide de perfusion d'un segment du tube digestif avec instillation en amont, aspiration en aval et utilisation d'un marqueur non absorbable pour calcul des débits. L'étude de patients présentant une stomie digestive permet des études sur plusieurs jours. La mesure des taux sanguins veineux post-absorptifs dans la circulation générale, est d'interprétation beaucoup plus difficile : un taux élevé peut refléter une absorption très efficace mais aussi une accumulation par non utilisation. Une mesure directe des concentrations d'acides aminés et du débit sanguin dans la veine porte, telle que celle réalisée par Rérat et al (1988) chez le porc, permet une mesure directe, tenant compte en plus du métabolisme intra-entérocytaire.

Le métabolisme peut également être étudié in vivo. L'utilisation des différentes catégories de nutriments peut être étudiée par calorimétrie indirecte avec mesure des dépenses énergétiques (consommation d'oxygène et production de gaz carbonique) couplée à la détermination du bilan azoté. L'utilisation de traceurs non radiactifs (isotopes stables) a représenté un progrès très important et a permis de mesurer directement synthèse et dégradation (Matthews et Bier, 1983). 
La première composante de ce progrès est d'ordre éthique : ces isotopes nonradioactifs sont sans danger et utilisables chez l'enfant. Le facteur limitant devient l'analyse par spectrométrie de masse et éventuellement le prix. À cet avantage, commun aux autres nutriments, s'ajoute celui spécifique aux protéines de l'intérêt du ${ }^{15} \mathrm{~N}$ puisqu'il n'y a pas d'isotope radioactif de l'azote à demi-vie longue.

\section{Que doit-on mesurer?}

II est possible de mesurer la balance azotée de manière globale : bilan azoté (entrées - pertes), rétention azotée, turn over protéique grâce à la ${ }^{13} \mathrm{C}$-Leu en cas d'utilisation d'isotopes stables.

Certains acides aminés ont des propriétés particulières et méritent d'être étudiés séparément, surtout dans des conditions pathologiques en nutrition thérapeutique : nutrition entérale par sonde digestive ou nutrition parentérale par voie intraveineuse. La glutamine en est un exemple. Cet acide aminé classiquement non essentiel va être métabolisé par l'entérocyte et permettre la synthèse d'autres acides aminés non essentiels tels l'alanine. Ceci est illustré dans le tableau IV. L'utilisation de la leucine, c'est-à-dire l'oxydation des protéines, n'est pas modifée en cas d'absence presque complète d'intestin grêle. Par contre, la consommation de glutamine est diminuée. La glutamine va aussi fournir de l'énergie et constituer une part importante du pool des acides aminés libres de l'organisme, en particulier au niveau musculaire. Cet acide aminé pose également des problèmes spécifiques : problèmes de stabilité, il n'est pas stable de manière durable en solution aqueuse et, de ce fait, manque dans les solutions d'acides aminés parentérales prêtes à l'emploi; ceci pourrait être résolu grâce à l'utilisation de peptides contenant de la glutamine qui, eux, sont stables. Des problèmes analytiques se posent également: I'hydrolyse acide qui permet de mesurer les aminogrammes des protéines ne la respecte pas et fait apparaître de l'acide glutamique. C'est pourquoi les données analytiques mentionnent habituellement la somme glutamine + acide glutamique. Nous avons donc besoin de méthodes de dosages et de données analytiques puisque nous ne connaissons même pas bien les quantités normalement ingérées et l'effet des traitements technologiques sur la teneur en glutamine des aliments.

La mesure de l'effet d'un apport protéique sur une fonction biologique est bien entendu l'idéal : ceci est aussi le plus difficile. L'alimentation est un tout, augmenter une classe de nutriments conduit à en baisser une autre; de plus, ration énergétique globale et turn over protéique sont liés.

\section{QUELQUES EXEMPLES PRATIQUES}

\section{Nourrisson}

C'est un marché important. C'est aussi une condition où les protéines laitières bovines

Tableau IV. Métabolisme des acides aminés. Effet de l'absence d'intestin (grêle inférieur à 1,5 $\mathrm{m})$.

Amino acid metabolism. Effect of absence of intestine (small bowel length $<1.5 \mathrm{~m}$ ).

Flux d'acides aminés ( $\mu$ mol. $\left.\mathrm{kg}^{-1} \cdot \mathrm{h}^{-1}\right)$ Leucine Glutamine

$\begin{array}{lll}\text { Témoins } & 83 & 348 \\ \text { Grêle court } & 85 & 279(P<0,05)\end{array}$

D'après Darmaun et al, 1988. 
peuvent être toxiques. Au cours de l'intolérance aux protéines du lait de vache, ces protéines se comportent comme des antigènes suscitant des réactions allergiques digestives ou générales. L'intestin est alors lésé, sa perméabilité aux protéines entières ou seulement partiellement dégradées est augmentée (Heyman et al, 1988). C'est un domaine oủ le fait de disposer de peptides suffisamment petits pour être moins immunogènes a constitué un progrès très important. Cette affection, dont la fréquence semble varier de 0,5 à $2 \%$, est caractéristique de la première année de vie.

\section{Nutrition médicalisée}

II s'agit de la nutrition de patients ne pouvant pas s'alimentr spontanément, ou nécessitant une alimentation différente par sa fréquence (continue) ou sa nature. Cette nutrition peut s'administrer par voie parentérale, intraveineuse, ou en utilisant la voie digestive, par voie orale ou par sonde digestive. Les protéines laitières sont largement utilisées.

L'importance de la forme administrée a fait l'objet de beaucoup de discussions. À un moment, il a semblé possible de proposer une combinaison synthétique d'acides aminés couvrant, au mieux, les besoins pour chacun d'entre eux. L'étude des anomalies congénitales du transport intestinal des acides aminés a conduit à réaliser qu'en fait, une bonne partie des acides aminés rentrent dans l'entérocyte sous forme de peptides. Ce n'est que dans la cellule qu'ils seront hydrolysés pour passer ensuite du côté sanguin. La plupart des solutions de nutrition proposent maintenant, soit des mélanges à base de protéines entières soit des peptides. Les mélanges à base de peptides semblent pouvoir être intéressants en cas d'atteinte du tube diges- tif avec diminution de ses capacités d'hydrolyse ou d'absorption (Cosnes et al, 1990) ou pour aider à récupérer d'une dénutrition aiguë (Poullain et al, 1989). L'importance respective de la qualité et de la quantité des protéines apportées reste l'objet de beaucoup de discussions. L'utilisation de peptides d'origine laitière peut avoir un autre avantage en permettant plus de flexibilité pour la formulation de nouveaux produits. Les aminogrammes et charges minérales associées peuvent être différents de ceux des protéines d'origine. L'ensemble de ces considérations a permis, par exemple, d'utiliser des peptides dont la production par hydrolyse a été mise au point dans le laboratoire de recherches de technologie laitière de I'INRA pour préparer des solutions entérales maintenant assez répandues (gamme Réabilan(\&). Une approche similaire est adoptée par de nombreuses sociétés.

\section{Populations particulières}

Une population particulière, déjà mentionnée, doit être individualisée : il s'agit des patients présentant une insuffisance rénale sévère mais dont la gravité ne nécessite pas encore le recours à la dialyse artificielle. Les protéines alimentaires mais aussi des composés associés tel le phosphore, largement présent dans le lait de vache, doivent être limités. Des études contrôlées récentes ont montré qu'une alimentation avec un apport très limité en protéines $(0,4 \mathrm{~g}$ de protéines par $\mathrm{kg}$ de poids corporel et par jour) pouvait ralentir la progression de l'insuffisance rénale (Ihle et al, 1989). Dans ce cas, la qualité des protéines ingérées est très importante. Une difficulté de ce régime est qu'il n'est pas appétissant. De plus, une restriction protéique sévère entraîne souvent une diminution de l'appétit, donc une diminution 
de l'apport calorique réel. Ce besoin de limiter l'apport protéique a donné naissance à de nouveaux produits (exemple : Alterna ${ }^{\circledR}$, de Ross laboratories, vendu aux Etats-Unis en tant que substitut du lait appauvri en protéines et en minéraux).

Le lait ne contient pas que des protéines. Le phosphore vient d'être mentionné. La présence de lactose peut également chez certains constituer un problème. En cas d'atteinte digestive sévère, l'activité de la lactase intestinale est souvent diminuée. L'étiquetage de beaucoup de produits de nutrition entérale mentionne donc qu'ils sont "sans lactose". En pratique, en France, il suffit que la quantité présente soit inférieure à 200 mg pour $100 \mathrm{~g}$ de produit. Une hypolactasie peut être également retrouvée chez des individus normaux et limiter l'ingestion de lait. Un traitement technologique particulier peut permettre de pallier cette insuffisance. Le cas du yaourt, forme qui permet une amélioration de la tolérance au lactose, est le plus connu (Kolars et al, 1984). Finalement, certains composants protéiques mineurs, acides aminés libres ou protéines peuvent au contraire être désirables en nutrition clinique.

\section{CONCLUSION}

En matière de protéines laitières, les médecins et les professionnels intervenant dans la nutrition clinique attendent beaucoup des industriels et des chercheurs. Idéalement, il faudrait pouvoir disposer de protéines et de peptides mieux caractérisés (exemple glutamine), de compositions variées. Plus l'effet des traitements technologiques sur leurs propriétés fonctionnelles et nutritionnelles seront connus, plus notre liberté d'action sera grande. Enfin, manger doît être agréable, ces pro- duits doivent avoir bon goût. Pour les peptides de petit poids moléculaire, ce dernier objectif, à lui seul, montre qu'il reste du travail.

\section{RÉFÉRENCES}

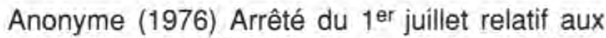
aliments diététiques et de régime de l'enfance. J Off Répub Fr (14 septembre 1976)

Anonyme (1990) Commission Interministérielle et Interprofessionnelle d'Étude des aliments destinés à une alimentation particulière : avis du 17 mai 1989 sur les mélanges nutritifs pour alimentation liquide spéciale. Bull Off Concurrence, Consom Répression Fraudes (2 février 1990)

Cosnes J, Evard D, Beaugerie L, Gobert JG, Le Quintrec $Y$ (1990) Prospective randomized trial comparing small peptides vs whole proteins in patients with a high jejunostomy. Clin Nutr 9 (suppl), 111 (abstr)

Darmaun D, Just B, Messing (1988) Glutamine kinetics in short-bowel patient. Gastroenterology 94 (suppl), A87 (Abstract)

Desjeux JF, Grasset E (1990) Spécificité de l'évaluation clinique des produits de nutrition. In: Pharmacologie Cliniques: Actualités et Perspectives (JP Poissel, C Caulin, M Teule, eds). Colloque INSERM/John Libbey Eurotext Ltd, Paris

Dupin H (1981) Apports nutritionnels conseillés pour la population française. Tech et Doc Lavoisier, Paris

Food and Drug Administration (1988) Nutrition labeling of food. In: 21 Code of Federal Regulations 101.9. US Gov Print Office, Washington

Heyman M, Grasset E, Ducroc R, Desjeux JF (1988) Antigen absorption by the jejunal epithelium of children with cow's milk allergy. Pediatr Res 24, 197-202

Kolars JC, Lewitt MD, Aouji M, Savaiano DA (1984) Yoghurt - an autodigesting source of lactose. $N$ Engl J Med 310, 1-3

Ihle BU, Becker GJ, Whitworth JA, Charlwood RA, Kincaid-Smith PS (1989) The effect of 
protein restriction on the progression of renal insufficiency. N Engl J Med 321, 1773-1777

Matthews DE, Bier DM (1983) Stable isotope methods for nutritional investigation. Annu Rev Nutr 3, 309-339

Monchi M, Vaugelade $\mathrm{P}$, Vaissade $\mathrm{P}$, Rérat A (1991) Net protein utilization after duodenal infusion of small peptides or free amino acids in growing rats. J Parenter Enterol Nutr (abstr) 15, 295

Munro HN, Crim MC (1988) The proteins and amino acids. In: Modern Nutrition in Health and Disease (ME Shils, VR Young, eds) Lea and Febiger, Philadelphia

National Research Council (US) Subcommittee on the Tenth Edition of the RDAs (1989) Re- commended Dietary Allowances. 10th edn. National Academy Press, Washington

OCDE (1990) Health Care Systems in Transition. The Search for Efficiency. OCDE, Paris

Poullain MG, Cézard JP, Roger L, Mendy F (1989) Effect of whey protein, their oligopeptide hydrolysates and free amino acid mixture on growth and nitrogen retention in fed and starved rats. J Parenter Enteral Nutr 13, 382386

Rérat $A$, Simoes Nuñes $C$, Mendy $F$, Roger $L$ (1988) Amino acid absorption and production of pancreatic hormones in non-anaesthetized pigs after duodenal infusions of a milk enzymic hydrolysate of free amino acids. $\mathrm{Br} J$ Nutr 60, 121-136 